\title{
Construction and Application of Intelligent Management of College Physical Education from the Perspective of New Science and Technology
}

\author{
Ren Huiyi ${ }^{1}$, Su Yingdong ${ }^{2}$ \\ ${ }^{1}$ Department of Physical Education, Jining University, Qufu, China \\ ${ }^{2}$ Jining Children and Women's Activity Center, Jining, China
}

Email address:

renhuiyi@sina.com (Ren Huiyi),jn3861@126.com (Su Yingdong)

\section{To cite this article:}

Ren Huiyi, Su Yingdong. Construction and Application of Intelligent Management of College Physical Education from the Perspective of New Science and Technology. Science Innovation. Vol. 9, No. 4, 2021, pp. 145-150. doi: 10.11648/j.si.20210904.15

Received: April 26, 2021; Accepted: May 19, 2021; Published: May 24, 2021

\begin{abstract}
Based on the in-depth study of the new science and technology involved in the university sports teaching management, this paper concludes that the focus of the contemporary university sports teaching management mode is sports teaching, evaluation and security, big data, artificial intelligence and other new technologies will become an important auxiliary means of university sports teaching management. This paper puts forward the basic framework and ideas of the application of new technology in the management of college physical education, designs the intelligent management mode of college physical education, compares it with the traditional management mode of physical education, and proves that the application of new technology can promote the scientization of physical education curriculum, improve the quality of college physical education, improve the quality of college physical education Under the basic principle of "student-centered", we should promote the implementation of "teaching students in accordance with their aptitude" in teaching content and "teaching methods vary from person to person", further improve the safety protection of curriculum construction and the rational use and development of sports resources, and promote the establishment of a comprehensive, scientific, fair and effective sports evaluation system for teachers and students.
\end{abstract}

Keywords: College, Physical Educationfrom, Intelligent, Management

\section{新科技视域下大学体育教学智能化管理的设想与构建}

任慧一 ${ }^{1}$, 苏迎冬 ${ }^{2}$

${ }^{1}$ 济宁学院体育系, 曲阜, 中国

2济宁市妇女儿童活动中心, 济宁, 中国

\section{邮箱}

renhuiyi@sina.com（任慧一），jn3861@126.com（苏迎冬）

摘要：本文通过对新科技介入大学体育教学管理进行了深入的研究得出：当代大学体育教学管理模式的重点是体育教 学、评价与安全, 大数据、人工智能等新科技必将成为大学体育教学管理的重要辅助手段。提出了大学体育教学管理 运用新科技的基本架构与思路, 设计了大学体育教学智能化管理的模式并与传统体育教学管理模式进行了对比, 论证 了新科技的应用能够在推进体育课程科学化、促进大学体育在“以学生为本”的基本原则下贯彻教学内容的“因材施教” 和教学方法的“因人而异”、进一步提升课程建设的安全防护和体育资源的合理利用与开发, 推动建立全面、科学、公 平、有效的师生体育评价体系等方面做出新的创新与改变。 
关键词: 大学, 体育教学, 智能化, 管理

\section{1. 引言}

随着时代的发展、“健康第一”的思想和人才培养的需 要, 国家对大学生身体素质极为关注。早在2002年, 教育 部就颁布了《全国普通高等学校体育课程教学指导纲要》, 《纲要》强调指出: “体育课程是大学生以身体练习为主 要手段, 通过合理的体育教育和科学的体育锻炼过程, 达 到增强体质、增进健康和提高体育素养为主要目标的公共 必修课程”, [1]因此, 如何合理的、有效的组织“身体练习” 是当前高校体育教学模式改革关注的重点, “不出汗、无 强度、无难度、无对抗”温柔体育被众人所诟病。2014年, 教育部又颁布了《高等学校体育工作基本标准》, 再次强 调“严格执行《全国普通高等学校体育课程教学指导纲 要》”, 并要求深入推进课程改革“每节体育课须保证一定 的运动强度, 其中提高学生心肺功能的锻炼内容不得少于 $30 \%$; 要将反映学生心肺功能的素质锻炼项目作为考试内 容。”[2]近年来, 青少年运动期间猝死现象频发, 体育锻 炼方式和手段的把握又成为了颇具争议的社会焦点, 使得 体育教师畏手畏脚, 学校左右为难, “降低强度、减少对 抗”等应付体育课的思想还是在一定程度上存在着, 这也 是长期困扰甚至阻碍大学体育教学发展的重要阻力。一边 强调要加强身体练习, 一边还要防范学生的安全问题, 如 何“鱼与熊掌兼得”, 从体育专业的角度来说, “运动强度” 的检测和控制是这两方面不能兼得的关键 [4]。就目前我国 高校体育课现有设备条件，对“运动强度”的把控是难以象 专业训练队伍一样实现的, 但随着当前新科技的高速发展, 对新科技介入体育教学进行一些理论探索和实践尝试是 当务之急。

\section{2. 新科技与大学体育教学管理的基础研究}

\section{1. 当前传统教学模式下大学体育教学管理中的一些问 题}

当前实行的大学传统体育教学模式在课程设置, 学生 选课, 兴趣提升, 教材优化, 考核标准等方面较比以往

的“以老师为主体”的体育教学模式有所改观, 逐渐提 高了大学体育课程“以学生为主体”的认识, 从普通教学转 向了选项课教学 [5]。但这种转变只是在授课时间, 课程项 目, 授课教师等方面为学生提供了“自选”模式, 在大部分 学生的体育兴趣爱好, 主动学习, 主动练习等方面的提升 尚未有真正的突破, 往往还是出现学生应付体育课, 不愿 意主动参加体育锻炼的现象, “运动强度的把控”是大学体 育教师难以逾越的障碍, 大学体育教学依然存在着“以教 师为主体” 的现象, 这些依然是困扰广大体育教学工作者 的棘手问题。因此, 如何实现“学生真正愿意学体育? 教 师真正认真教体育? 学生真正科学练体育? 获得科学地 体育评价反馈? ”等现实问题应成为当前大学体育教学研 究的重点之一。

\section{2. 新科技成为大学体育教学管理重要辅助手段的可行 性}

2018年, 湖北潜江返湾湖湿地国际马拉松赛事充分利 用图像识别、数据跟踪、紧急救护和GIS卫星定位等诸多 智能技术手段, 实时监测运动员安全、实时观看参赛视频、 科学调度安保、医疗、交通等, 极大程度提高了赛事运营 的效率。[6]通过新科技介入体育产生的多方尝试经验, 从 理论应用上来说, 利用新科技模拟人类智能行为的基本理 论、方法和技术是完全能够大幅度提升体育教学过程实施 的质量、进度和效率; 同时, 可以通过结合初始数据分析 和数据跟踪进行分析, 形成一套全面、详实、科学地评价 系统。具体的设想是, 例如有希望改变体育课程中“教学、 兴趣、安全、有效”的融合问题, 通过对学生的初始及运 动身体数据的大数据技术分析, 可以有效帮助教师预防因 个别学生不健康的生活习惯, 亚健康的身体状况以及长期 的疾病影响下运动强度的把控, 并借助学生心率采集和数 据分析, 在安全的前提下提升学生的有效运动强度并形成 科学的身体练习进阶及客观评价反馈等。但是, 这还需要 体育专业与计算机专业的跨学科联合, 从基础理论构建到 技术对接, 转变传统观念, 共同实践“新科技+体育教学” 设计理念。

\section{3. 新科技在体育教学管理应用中的困难}

在投入上, 新科技在大学体育教学建设中需要一定 的设备和人力投入, 增加了学校或者教育主管部门的财 政开支和人力资源; 新科技开发的平台建设还需要大量 数据采集的支撑和效验, 在时间和空间上也会造成一些 人力、物力和财力的支出等。不过, 当前国家推动智慧 城市、智慧校园建设, 新科技在体育教学中的介入, 可 以提升体育教学的智能化、智慧化, 这也是助力智慧校 园建设的有力举措。[9]在思想上, 部分教师“冬练三九, 夏练三伏”的传统体育“苦练”的思想根深蒂固, 对新科技 介入体育训练或者体育教学存在一定的质疑; 部分体育 工作者对人工智能平台介入的有效性和科学性还有一定 的顾虑; 大数据对体育数据分析的深度与广度, 还尚需 一定的时间进行效验等。不过, 新科技在体育教学中的 应用是顺应当前科技引领教育发展的大趋势, 减少经验 体育思维, 转变科技可以有效提升运动训练效果的观念, 提高以数据分析为体育教学管理及评价主体建设的科学 理念, 有助于有效地增强大学生体质、科学地增进大学 生健康和不断提高大学生的核心素养, 这也是圆满完成 《全国普通高等学校体育课程教学指导纲要》主要目标 的重要抓手。 


\section{3. 大学体育教学智能化管理的基本架构与思路}

\section{1. 大学体育教学智能化管理的基本架构}

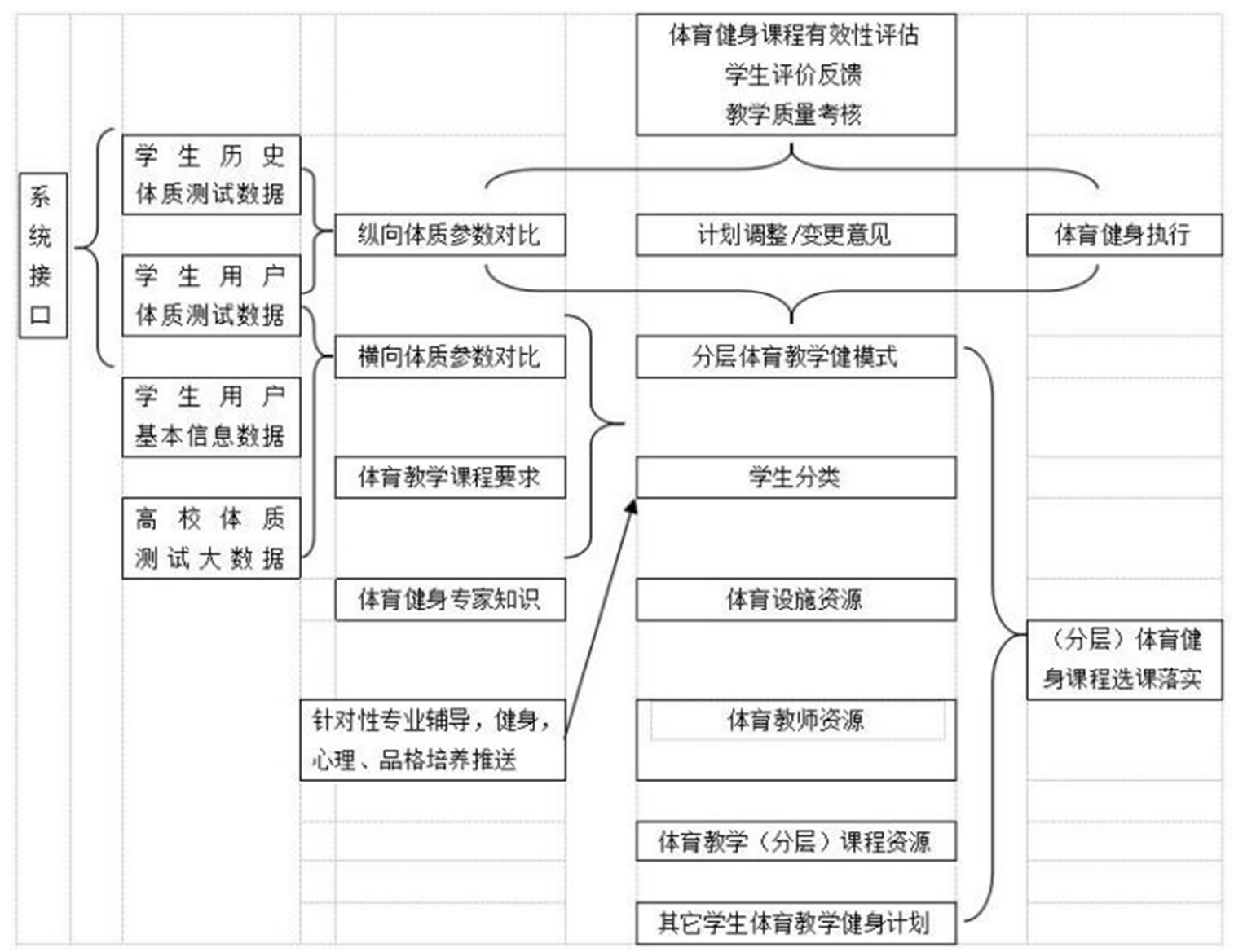

图1 大学体育教学智能化管理的基本架构。

\section{2. 大学体育教学智能化管理的基本思路}

大学体育教学智能化管理的设计基本思路是以人为 本、科技助推体育发展, 利用新科技来促进高校体育教育 的改革发展的主导思想。在体育教学上, 以学校体育分层 教学模式为基本教学基础, 从师生的角度出发, 结合实际 情况, 以大学生体育需求为依据, 以静态心率和运动心率 预测和规划运动强度, 从生理、心理两个方面对大学生体 育教学推送合理化方案和建议, 贯彻和执行“健身性与文 化性相结合”、“选择性与实效性相结合”、“科学性和可接 受性相结合”、“民族性与世界性相结合”的《全国普通高 等学校体育课程教学指导纲要》体育课程教学原则。[1] 通过学生自评、互评和教师评定等方式, 结合人工智能科 学数据反馈, 并通过大学生的体能与运动技能、认知、学 习态度与行为、交往与合作精神、情感表现等确定总体评 价框架、目标和体系。在系统构建上, 体育专业总体规划, 确保系统的高度集成, 安全可靠, 突出实用性; 计算机专 业具体规划, 分布进行设计, 确保在总体上把握全局, 减 少疏漏; 在进行平台软、硬件配置和栏目的设置上, 尽量 考虑采用较为先进的技术, 确保所设计的管理系统能够满
足体育教学的需求, 并具有一定的先进性和可扩展性, 平 台框架内部是由多个管理系统模块组成的, 这些模块通过 人工智能的技术形成密切的相互耦合, 增强模块之间的粘 性。[10]

\section{4. 大学体育智能化教学管理的成效}

\section{1. 新旧体育教学模式的功能对比}

上世纪, 我国大学体育教学常采取“早操+大学体育课 +课外体育活动”的形式 [11], 在本文中暂称之为“传统体育 教学模式”; 2000年以来, 不少高校在探索和尝试体育教 育的新模式, 例如“三自主”的公共体育教学, 开展体育第 二课堂、推行俱乐部会员制、甚至建立跟踪大学生锻炼的 APP等, 本文暂称为“现行体育教学模式”。本文系统的理 论构建是以学校体育分层教学模式为理论基础, 依靠新科 技等先进科技手段, 完成必修考核、身体达标和科学健身 指导等项目的综合检测、分析与评价, 现简称为“体育教 学智能化管理模式”。 
图2 新旧体育教学模式功能对比一览表。

( ○: 无 $\sqrt{ }$ : 有)

\begin{tabular}{llll}
\hline 功能 & 传统体育教学模式 & 现行体育教学模式 & 体育教学智能化管理模式 \\
\hline 入学体检档案管理系统 & $\circ$ & $\circ$ & $\sqrt{ }$ \\
入学身体机能评价 & $\circ$ & $\circ$ & $\sqrt{ }$ \\
入学体测数据采集 & $\sqrt{ }$ & $\sqrt{ }$ & $\sqrt{ }$ \\
入学体测评价 & $\circ$ & $\circ$ & $\sqrt{ }$ \\
静态心率分析 & $\circ$ & $\circ$ & $\sqrt{ }$ \\
课堂心率监测预警 & $\circ$ & $\circ$ & $\sqrt{ }$ \\
运动强度预警 & $\circ$ & $\circ$ & $\sqrt{ }$ \\
身体安全预警提示 & $\circ$ & $\circ$ & $\sqrt{ }$ \\
个人疾患智能辅助 (自愿) & $\circ$ & $\circ$ & $\sqrt{ }$ \\
体育健身项目智能规划 & $\circ$ & $\circ$ & $\sqrt{ }$ \\
心理测评 & $\circ$ & $\circ$ & $\sqrt{ }$ \\
心理健康评价 & $\circ$ & $\circ$ & $\sqrt{ }$ \\
心理健康教育推送 & $\circ$ & $\circ$ & $\sqrt{ }$ \\
体育健康规划 & $\circ$ & $\circ$ & $\sqrt{ }$ \\
自主选课系统 & $\circ$ & $\sqrt{ }$ & $\sqrt{ }$ \\
智能优化选课 & $\circ$ & $\circ$ & $\sqrt{ }$ \\
外堂技能课教学 & $\circ$ & $\sqrt{ }$ & $\sqrt{ }$ \\
网络视频教学 & $\sqrt{ }$ & $\sqrt{ }$ & $\sqrt{ }$ \\
理论知识测试 & $\circ$ & $\sqrt{ }$ & $\sqrt{ }$ \\
品格培养学习 & $\circ$ & $\circ$ & $\sqrt{ }$ \\
教师教学资源管理 & $\circ$ & $\circ$ & $\sqrt{ }$ \\
课外体育指导 & $\circ$ & $\sqrt{ }$ & $\sqrt{ }$ \\
健康及品格综合评价 & $\circ$ & $\circ$ & 云端 \\
教师综合评价 & $\circ$ & $\circ$ & 计算机 \\
数据存储方式 & $\circ$ & $\circ$ &
\end{tabular}

\section{2. 新科技解决体育教学管理的实际问题}

大学生体育教学智能化管理由多个模块组成, 但其中 体质跟踪采集, 运动强度的预警与规划, 数据分布比较, 体育教学的反馈与评价, 学生兴趣爱好、身心健康、品格 培养的效果及评价反馈等 4 个主要基本功能模块。在功能 模块的开发、对接和运行不是难题, 但模块之间的粘性却 需要体育各学科间基础理论的支撑和体育教学原则的执 行, 这是该教学管理应用的重点和难点。

\subsection{1. 体质跟踪采集}

体质跟踪采集包括了参与大学生入学前体质数据录 入, 体育课程中“心率数据采集, 运动强度数据监控, 进 阶式体质数据采集”[13]等几个方面, 主要的目的是对参与 大学生有一个较为全面的身体基本机能的数据分析和预 测, 并通过人工智能技术协助和规划大学生体育课的项目 选择、课程个性化, 运动强度有效范围, 安全防护预警设 置等, 并辅助教师掌握课上教学和训练的效果, 及时把控 学生的运动强度等。

\subsection{2. 预警保护功能}

通过对参与大学生体育跟踪采集, 以及平时体育课上 的数据反馈, 并结合人体心肺功能的大数据比对, 为每个 学生预测和制定有效强度的心率值范围。通过终端设备反 馈给授课教师, 在科学完成体育练习的同时做好大学生的 安全预警和保护, 一方面提高大学生高强度训练时身体机 能的安全防护, 另一方面也可以缓解教师因为安全问题不 敢对参与大学生实施有效运动强度训练的心理负担。

\subsection{3. 数据分布比较}

基于高校学生体质数据库, 以及参与大学生体质测试 数据, 体育兴趣及心理特征等进行人工智能分析后, 可以 有以下几个方面的数据分布比较, 为后续的体育课程设置, 体育资源开发和体育科研工作提供一定的帮助: (1)横向比 较。大学生体质参数与高校群体体质参数分布比较; 大学 生兴趣爱好与高校兴趣爱好参数分布比较。(2)纵向比较。 大学生体质参数历史比较, 大学生体育学习及历史成绩比 较。(3)大数据分类。通过大数据对参与大学生进行体质参 数分类, 运动强度分类, 兴趣爱好分类, 心理特征分类, 学习效果分类等。

\subsection{4. 学生兴趣爱好、身心健康、品格培养的效果及评价 反馈}

首先, 从大学生入学的身体素质、心理、专业等数据 开始着手建立基础数据库, 同时引入大数据分析, 建立初 始基本评价体系; 再者, 为每位大学生进行人性化的体育 教教学和健身进行规划和定制, 根据自身的兴趣、爱好和 专业等特点对体育课选项进行人工智能优化推荐, 实现体 育网上教学与外堂课实践相结合, 同时增加课外时间运动 跟踪, 科学健身理论的网上学习、心理健康指导、品格培 养等; 最后, 分列出大学生每年的教学、健身的完成情况, 身体指标、健康指数及品格培养目标, 成为完成必修考核 的依据, 并形成教学与健身, 兴趣与品格, 线上与线下的 综合评测体系, 真正实现“学生愿意学体育、教师认真教 体育、学生科学练体育、科学地进行体育综合评价”。 


\section{5. 结论}

在当代新科技迅猛发展的趋势下, 新科技为世界带来 的高效率和高成效显而易见。在大学体育教学管理方面, 固有传统体育教学管理模式的经验和体系已经出现了掣 肘和瓶颈, 大学体育教学管理模式亟待创新。鉴于此, 本 文通过相关的基础研究对传统体育教学模式的现状及问 题进行了探讨, 并初步尝试提出智能化教学管理的基本架 构与思路, 论证了智能化教学管理的科学性与创新性。再 者, 当代大学体育教学管理模式的重点是体育教学、评价 与安全。大学生身体素质的提高, 体育课肩负着重要的责 任, 上好体育课, 体育教学责无旁贷。体育教学过程是指 “体育教学实施、运行的时空连续过程。作为一种系统运 行工程, 是师生共同参与的, 通过确定目标、激发动机、 理解内容、进行操作、反馈调控、评价结果等环节组成”。 [15]因此, 如何把这些方面有机的结合起来, 仅凭人工操 作的体育课已经很难获得良好的效果。通过以上的论述, 可以清晰地认识到人工智能技术及大数据分析的介入, 首 先是提升了体育教学的工作效率, 这种大量的体育数据采 集及分析运算, 是往年人工采集和分析难以企及的工作。 再者, 把体育教学的“目标”、“动机”、“内容”、“操作”、“调 控”、“评价”有机的结合起来, 粘在一起, 真正的实现“因 材施教”, 这也是以往的体育教学模式难以实现的; 还有, 安全问题无小事, 每个大学生体育锻炼的安全问题不仅关 系到个人, 还关系到家庭的和睦和社会的安定。以往体育 教师因学生的安全问题而降低体育课的运动强度、技术难 度是可以理解的, 因为体育教师没有可以把控每个孩子运 动强度的依据和保障。人工智能技术的介入大大缓解了体 育教师的这种顾虑, 从个体体质健康数据一心率跟踪 一运动强度规划, 基本可以做到为学生“量体”定制教学 任务, 为体育教师制定“因人而异”的教学计划, 让运动强 度最安全, 运动风险降到最低, 保护了学生的同时也保护 了教师。随着人工智能逐渐深入到人们的生活中,

“新科技+体育”势必是未来助推体育发展的主动力。 全国政协委员、北京体育大学副校长胡扬委员就指出: “人 工智能可以极大地提升大众参与健身的体验度和健身效 果, 有效帮助大家了解健身效果。同时, 随着大众在健身 活动中使用智能设备, 将会产生大量的个人健身数据、健 康数据。而这些数据恰恰可以对我国国民体质现状评估, 以及体育政策的制定有重要的参考意义。”从“新技术+体 育教学”的理论探索和架构设想来看, 人工智能技术在体 育教学中的应用能够在推进课程科学化, 实现教学内容的 “因材施教”和教学方法的“因人而异”，提升课程建设与资 源开发, 建立全面、科学、公平、有效的师生体育评价等 方面做出新的创新与改变。

\section{致谢}

感谢济宁市政府、济宁学院、山东省教育科学规划领 导小组办公室、山东省社会科学界联合会对青少年体育与 健康的高度关注, 以及对青少年体育教学数字化建设和智 能化改革的支持。上述单位分别于2019年批准“基于人工 智能的青少年体育教育健身服务云平台”(2019ZDGH032)
为济宁市重点研发计划项目立项, 济宁学院“课程思政”示 范课程建设项目立项（KCSZ201926），“基于人工智能的 山东省大学体育教学管理及评价系统建设研 究”(BYZN201909)为山东省教育科学“十三五”规划项目立 项；2020年批准“智慧校园建设背景下山东省学校体育数 字化模式发展研究”（20CSDJ07）为山东省社会科学规划 重点项目立项。鉴于政府、学校和社会各界的信任与托付, 经过广泛的资料收集与整理, 理论研究与论证, 项目测试 与实践, 在智能化体育教学应用和数字化教学模式的构建 等方面初步形成了一定的阶段性成果。在此研究基础之上, 秉承持之以恒的精神, 理论付之于应用的目标, 冀望在青 少年体育与健康的科学发展之路上再助一臂之力。

\section{参考文献}

[1] 教育部.教育部关于印发《全国普通高等学校体育课程教学 指导纲要》的通知 [EB/OL].http://www.moe.gov.cn/s78/A17/twys_left/moe_938 /moe_792/s3273/201001/t20100128_80824.html, 2002-8-6.

[2] 教育部.教育部关于印发《高等学校体育工作基本标准》的 通

[EB/OL].http://www.moe.gov.cn/srcsite/A17/moe_938/s3273/ 201406/t20140612_171180.html, 2014-6-12.

[3] 新 一 代人工智能发展规划 [EB/OL].http://www.gov.cn/zhengce/content/2017-07/20/cont ent_5211996.htm,2017-8-15.

[4] Ozbey N, Karakose M, Ucar A. The determination and analysis of factors affecting to student learning by artificial intelligence in higher education[C]//nformation Technology Based Higher Education and Training (ITHET), 2016 15th International Conference on. IEEE,2016:1-6.

[5] 杨异.网络时代下提高大学生体育兴趣教学方式的研究 [J]. 当代体育科技,2017(18):100-102.

[6] 刘佳吴.网络与数字时代的体育产业 [J]. 体育科 学,2019,39(10):56-64.

[7] 教育信息化十年发展规划（2011-2020 年） [EB/OL].http://old.moe.gov.cn//publicfiles/business/htmlfiles/ moe/s5892/201203/xxgk_133322.html,2012-03-13.

[8] 郑芳,徐伟康.我国智能体育:兴起、发展与对策研究 [J].体育 科学, 2019,39 (12) : 14-24.

[9] 王运武,于长虹.智慧校园:实现智慧教育的必由之路[M].北 京: 北京电子工业出版社,2016:8.

[10] Barnes T, Boyer K, Sharon I, et al. Preface for the Special Issue on AI-Supported Education in Computer Science[J]. International Journal of Artificial Intelligence in Education, 2017(1):1-4.

[11] 王广磊,李晓静,宋旭,滚军军.信息化背景下大学体育教学方 式变革研究[J].山东体育学院学报,2018(1):134-136. 
[12] 苏迎冬,冯吴暄,任慧一.校园跑步打卡类APP软件在高校体 育教学中的应用前景与发展趋势 [J]. 济宁学院学 报,2020(2):115-1119.

[13] Roll I,Wylie R.Evolution and revolution in artificial intelligence in education[J].International Journal of Artificial Intelligence in Education,2016(2):582-599.

[14] 刘东旭,李佳怡.人工智能与学校体育深度融合的理论研究 [C].Proceedings of 2019 5th International Conference on Humanities and Social Science Research (ICHSSR 2019) (Advances in Social Science, Education and Humanities Research, VOL.319)。
[15] 刘善言.学校体育学[M].北京: 中国教育出版社, 2006: 46 .

\section{作者简介}

任慧一(1974-), 男, 山东济宁人, 济宁学院体育系副教 授, 硕士, 研究方向: 体育人文社会学。

苏迎冬(1974-), 女, 山东济宁人, 济宁市妇女儿童活动 中心副研究馆员, 研究方向: 教育学。 